\title{
The Relationship of Insulin Resistance and Polycystic Ovary Syndrome: Effects of Metformin Therapy and ovarian Drilling
}

\author{
Asmaa F Hassan, Omyma G Ahmed, Marwa A Ahmed \\ and Hosam O Hamed*, \\ Departments of Physiology and Obstetrics \& Gynecology* \\ Faculty of Medicine, Assiut University
}

\begin{abstract}
Background: Polycystic ovary syndrome (PCOS) is a complex reproductive endocrine-metabolic disorder and insulin resistance (IR) is a common feature of it. Objective: To clarify the pathophysiological mechanisms of POCS, highlight its basic relationship with IR and to investigate the efficacy of metformin therapy and ovarian drilling in these cases. Patients and Methods: Thirty two PCOS patients (sixteen received metformin $850 \mathrm{mg}$ twice daily for four months[group 1] and sixteen underwent laparoscopic ovarian drilling [group 2]) and fifteen healthy controls were included in this study. Body mass index (BMI) evaluation followed by ultrasound examination for measurement of antral follicles count (AFC) in both ovaries, oral glucose tolerance curve, fasting blood glucose, insulin and glucose / insulin ratio (G/I) were measured in addition to serum total testosterone (T), leutinizing hormone (LH) and insulin like growth factor-1 (IGF-1) for all subjects. These parameters were reevaluated again 4 months after either types of treatment. Results: PCOS patients had significant increase BMI, T, LH, IGF-1, fasting blood glucose, insulin level and AFC but G/I ratio was significantly lower. Significant negative correlations between fasting G/I ratio and either of BMI, T, and AFC respectively were evident. Impaired fasting glucose level at baseline was observed in PCOS patients while after metformin and laparoscopic drilling it was significantly improved. Metformin significantly decreased BMI, serum T, LH levels, IGF-1 and AFC and increased the G/I ratio versus pretreatment values. Also, ovarian drilling has similar but less obvious effects. Conclusion: IR plays a vital pathophysiological role in PCOS patients as manifested by causal relationship between insulin resistance and metabolic and reproductive changes of PCOS. Metformin and ovarian drilling potentially improve these changes.
\end{abstract}

\section{INTRODUCTION}

PCOS is a complex endocrinemetabolic disorder characterized by chronic anovulation and/or clinical/ biochemical hyperandrogenism and/or polycystic ovaries

ultrasonography ${ }^{(\mathbf{1})}$. It is an

endocrinological disorders affecting $6-10 \%$ of females of reproductive $\operatorname{age}^{(2,3)}$

IR is a common feature of PCOS and is more in obese women, suggesting that PCOS and obesity have a synergistic effect on the magnitude of the insulin disorder. It 
leads to increased insulin secretion by beta-cells and compensatory hyperinsulinemia $^{(4)}$. IR in PCOS was considered a risk factor for gestational diabetes $^{(\mathbf{5})}$. Moreover, If beta-cell compensatory response declines, relative or absolute insulin insufficiency develops which may lead to glucose intolerance and type 2 diabetes $^{(6)}$.

On the basis of the theory that IR and hyperinsulinemia may be a relevant contributor to the pathophysiology of PCOS, it has been hypothesized that insulin-lowering agents (metformin), by reducing hyperinsulinemia, might improve the metabolic and reproductive abnormalities of $\operatorname{PCOS}^{(7,8)}$. Nevertheless, the mechanisms underlying the beneficial effects of metformin in the treatment of PCOS remain debated $^{(\mathbf{9})}$. Besides, laparoscopic ovarian drilling was found to have significant reproductive and metabolic effects in women with hyperinsulinemia ${ }^{(\mathbf{1 0})}$. This study aimed to clarify the pathophysiological mechanisms of PCOS and highlight on the basic relationship between it and IR and to investigate the efficacy of metformin therapy and ovarian drilling in women with PCOS and insulin resistance.

\section{PATIENTS \& METHODS}

\section{Study population:}

Thirty two PCOS patients with age ranged between 22-35 years (16 patients received metformin $850 \mathrm{mg}$ twice daily for four months (according to Trolle et al. ${ }^{(11)}$, group 1 , and(16 underwent laparoscopic ovarian drilling, group 2, (4- 8 punctures, each for 4 seconds according to ovarian volume) using monopolar diathermy (adjusted to 40- 60 watt), were included in the study. Patients were resistant to Clomiphene citrate (selective estrogen receptor modulator used mainly in female infertility due to anovulation) and they were recruited from Gynecology outpatient clinics in cooperation of Gynecology \& Obstetrics and Physiology Departments, Faculty of Medicine, Assiut University. They all met the criteria for diagnosis of PCOS according to Rotterdam Consensus $(2004)^{(\mathbf{1 2})}$. The diagnosis requires 2 of the following 3 criteria:

1-Amenorrhea or chronic oligomenorrhea ( $\leq 6$ menstrual cycle/year) indicating chronic ovulatory disorder.

2-Clinical (hirsutism and /or acne) or biochemical hyperandrogenism after exclusion of other causes of hyperandrogenism.

3-Polycystic ovarian morphology by vaginal ultrasound examination.

Fifteen age matched healthy controls have body mass index (BMI) less than $25 \mathrm{~kg} / \mathrm{m}^{2}$, regular menstrual cycles and normal ultrasound examination of the ovaries with no clinical or biochemical signs of hyperandrogenism were recruited from outpatient clinics for routine check-up purpose. The study was approved by the Assiut University Ethics Committee and every participant signed the informed consent before enrollment.

Exclusion criteria: pregnancy, use of hormones or hormonal contraception, for at least 3 months prior to the study, renal, hepatic or endocrinal 
disorders and family history of diabetes.

For both control and patients groups physical examination and BMI (body weight $(\mathrm{Kg})$ divided by height (meters squared) ${ }^{(\mathbf{1 3})}$ were done followed by ultrasound examination for counting of total antral follicles count (AFC) in both ovaries.

\section{Labratory assay:}

- In the same day a basic oral glucose tolerance test (OGTT) was done at $08.00-10.00 \mathrm{AM}$ after overnight fasting (10-14 h). The fasting glucose sample followed by another 4 samples was taken at $30,60,90$, and 120 minutes after giving them $75 \mathrm{gm}$ oral glucose load.

- The serum was analyzed for glucose and insulin immediately following separation from blood and the remainder of serum was stored at $-20^{\circ} \mathrm{C}$ for later uses. Serum glucose was measured by Hexokinase enzymatic method and spectrophotometric quantitation (Glucose kit, Sigma). Fasting insulin was measured by using (UBI- MAGIWEL insulin quantitative kit by ELISA method). Glucose tolerance was assessed by The 1997 American Diabetes Association and 1999 World Health Organization Criteria $^{(14)}$

- Fasting blood sample was taken for assaying total testosterone (T) (FERFTIGNX-T-kit), Leutinzing Hormone (LH) (DRG diagnostic kit), and Insulin like Growth Factor-1 (IGF-1) (immunodiagnostic system limited, UK, kit) by ELISA method.
- According to the basal levels of fasting glucose and insulin, PCOS patients were considered to be IR as fasting Glucose/Insulin (G/I) ratio less than 4.5 and/or fasting insulin level more than $20 \mathrm{u}$ $\mathrm{IU} / \mathrm{ml}^{(15)}$.

- All samples were taken in the first week of a spontaneous menstrual cycle (patients and controls) or progesterone withdrawal bleeding (patients).

- All previous parameters were reevaluated again 4 months after receiving of each treatment modality.

\section{Statistical analysis}

Data are expressed as mean $\pm \mathrm{SE}$ for all parameters. The data were analyzed by using GraphPad Prism data analysis program (GraphPad Software, Inc., San Diego, CA, USA). For the comparison of statistical significance between patients and normal subjects, or in patients before and after therapies Student NewmanKeuls t-test for unpaired and paired data were used. For multiple comparisons, one-way analysis of variance (ONE- WAY-ANOVA) test followed by least Significant Difference (LST) was used. Correlations were assessed using Spearman's non-parametric correlation coefficient $\rho$ according to that described by Knapp and Miller, (16). $\mathrm{P} \leq 0.05$ was considered statistically significant.

\section{RESULTS}
1.Clinical, metabolic and reproductive characteristics of women with PCOS at baseline (Table 1): The PCOS patients 
group had significant increase BMI, T, LH, IGF-1, fasting blood glucose and insulin levels and AFC while decrease $\mathrm{G} / \mathrm{I}$ ratio compared with control group $(\mathrm{P}<0.001)$.

2. Correlation and linear regression (Figure 1a, b, and c): At baseline evaluation of PCOS patients, there was significant negative correlation between fasting $\mathrm{G} / \mathrm{I}$ ratio and $\mathrm{BMI}$ $(\mathrm{r}=-0.71, \mathrm{P}<0.001), \mathrm{T}(\mathrm{r}-0.74, \mathrm{P}$ $<0.001)$ and AFC $(\mathrm{r}=-0.78$, $\mathrm{P}<0.001)$ respectively.

3. Alteration of OGTT in PCOS patients and controls after therapies (Figure 2 a, b and c): In OGTT, PCOS patients had impaired fasting glucose $(110-126 \mathrm{mg} / \mathrm{dl})$ at baseline when compared with controls (Fig. 2 a). Administration of metformin in group (1) and laparoscopic ovarian drilling in group (2) significantly lowered the blood glucose level compared with pretreatment values 3 .

4. Effect of metformin and ovarian drilling on metabolic and reproductive parameters in $\mathrm{PCOS}$ patients (Table 2): Administration of metformin significantly decreased BMI, serum T, LH levels, IGF-1 and AFC $(\mathrm{P} \leq 0.001)$ and increased the $G / I$ ratio $(P \leq 0.001)$ compared with pretreatment values. In addition, ovarian drilling significantly decreased serum $\mathrm{T}$ and AFC, IGF-1 $(\mathrm{P} \leq 0.001)$ and $\mathrm{LH}$ levels $\quad((\mathrm{P} \leq 0.01), \quad$ significant increase $\mathrm{G} / \mathrm{I}$ ratio $(\mathrm{P} \leq 0.05)$ and had no significant effect on BMI compared with preoperative values.

Table (1): Clinical characteristics and hormonal analysis of PCOS women at baseline

\begin{tabular}{lll}
\hline & $\begin{array}{l}\text { Controls } \\
(\mathbf{n}=\mathbf{1 5})\end{array}$ & $\begin{array}{l}\text { PCOS } \\
(\mathbf{n}=\mathbf{3 2})\end{array}$ \\
\hline Age (year) & $28.6 \pm 2.6$ & $29.3 \pm 4.5 \mathrm{NS}$ \\
BMI (kg/m2) & $23.5 \pm 2.2$ & $36.2 \pm 3.4 * * *$ \\
Testost (ng/dl) & $42.5 \pm 8.7$ & $97.6 \pm 15.2 * * *$ \\
LH( IU/l) & $5.6 \pm 1.2$ & $15.2 \pm 2.6 * * *$ \\
IGF-1 (ug/l) & $118 \pm 29.5$ & $187 \pm 35.6^{* * *}$ \\
F. glucose (mg/d) & $86 \pm 11.3$ & $119 \pm 20.2^{* * *}$ \\
F.insulin (uIU/ ml) & $12 \pm 3.2$ & $35.5 \pm 12.6 * * *$ \\
F. G/I ratio & $7.53 \pm 0.8$ & $3.51 \pm 0.6^{* * *}$ \\
AFC & $8.2 \pm 2.4$ & $33.9 \pm 11.85^{* * *}$ \\
\hline
\end{tabular}

NS: Non Significant, $* * * \mathrm{P} \leq 0.001$ (Patient vs. Control), Testost: Testosterone,

BMI: Body Mass Index, LH: Leutinizing Hormone IGF-1: Insulin Growth Factor-1, F: Fasting, G/I: Glucose/Insulin AFC: Antral Follicular Count 
Table (2): Comparison of clinical characteristics, hormonal analysis and AFC in PCOS women before and after metformin therapy and ovarian drilling

(Values are mean \pm SE)

\begin{tabular}{lllll}
\hline & Metformin & \multicolumn{2}{l}{ Ovarian drilling } \\
\cline { 2 - 5 } & Before & After & Before & After \\
\hline F. G/I ratio & $3.33 \pm 0.4$ & $6.76 \pm 0.6 * * *(103 \%)$ & $3.4 \pm 0.6$ & $4.2 \pm 0.8 *(24 \%)$ \\
Testost (ng/dl) & $95.7 \pm 13.5$ & $55.3 \pm 9.1 * * *(42 \%)$ & $96.6 \pm 14.1$ & $60.0 \pm 10.2 * * *(38 \%)$ \\
LH ( IU/I) & $14.88 \pm 0.26$ & $11.08 \pm 0.37 * * *(26 \%)$ & $15.86 \pm 0.27$ & $14.74 \pm 0.2 * *(7 \%)$ \\
IGF-1(ug/l) & $187.7 \pm 34.7$ & $162.5 \pm 27.7 * * *(13 \%)$ & $189.8 \pm 30.7$ & $177.1 \pm 20.5 * * *(7 \%)$ \\
BMI (kg/m2) & $35.9 \pm 4.4$ & $28.5 \pm 3.7 * * *(21 \%)$ & $36.1 \pm 3.6$ & $34.1 \pm 3.9 \mathrm{NS}(6 \%)$ \\
AFC & $32.6 \pm 5.5$ & $16.2 \pm 3.8 * * *(50 \%)$ & $30.9 \pm 3.4$ & $14.3 \pm 3.1 * * *(54 \%)$ \\
\hline
\end{tabular}

NS: Non Significant, BMI: Body Mass Index, LH: Leutinizing Hormone, IGF-1: Insulin Growth Factor-1, F: Fasting, G/I: Glucose/Insulin ratio AFC: Antral Follicular, Count Testost: Testosterone $\%=\%$ change value after versus before.

$* * *=\mathrm{P} \leq 0.001, * *=\mathrm{P} \leq 0.01, *=\mathrm{P} \leq 0.05$, Significance of values after Vs before.
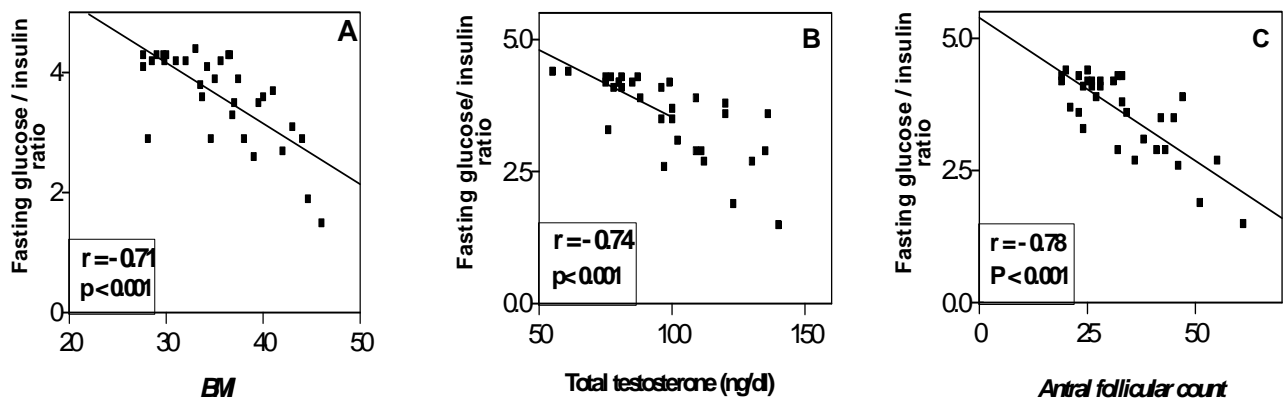

Fig.(1): Correlation and linear regression between fasting G/I ratio and BMI (A), total testosterone (B) and AFC(antral follicular count) (C) in PCOS patients at base line. 


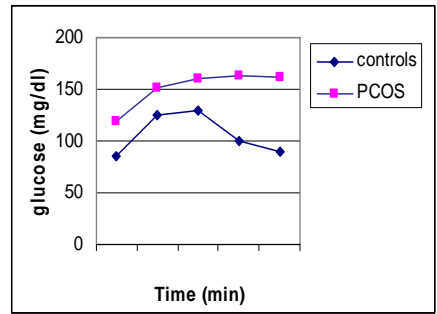

Fig (2a): OGTT in control and PCOS and PCOS patients at Base line $(\mathrm{P}<0.001)$ at fasting 30 , $60,90,120$ minutes as compared to control.

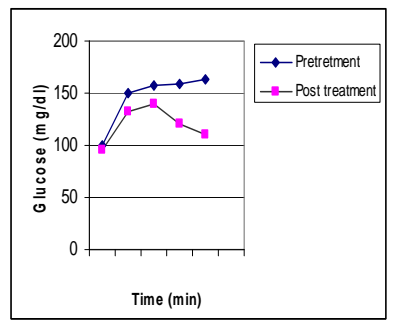

Fig (2b): OGTT before and after Metformin treatment in PCOS ( $p$ is non significant at fasting, $\mathrm{P}<0.01$ at $30,60,90$ and 120 minutes.

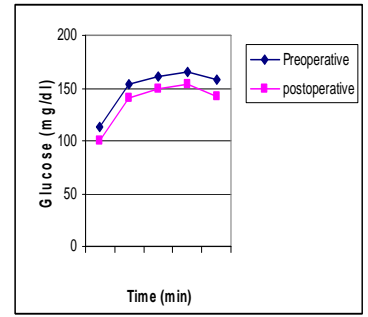

Fig (2c): OGTT before and after Ovarian drilling in PCOS Patients ( $\mathrm{P}$ is non significant at fasting, $\mathrm{P}<0.05$ at $30,60,90$ and 120 minutes.

Fig (2): Oral glucose tolerance curve (OGTT) in control and PCOS patients before and after ovarian drilling Metformin treatment

\section{DISCUSSION}

Insulin resistance is defined as the inability of insulin to exert its physiological effect. It is manifested peripherally (at the tissues) or centrally (at the liver) through reduction in the ability of insulin to lower plasma glucose ${ }^{(17)}$. The pathophysiology of PCOS is still largely unknown and until now, medical care of these subjects has been limited to symptomatic control and infertility ${ }^{(\mathbf{1 8})}$. While the basic pathophysiological mechanisms seem to be caused by dysfunction of the ovary, the clinical expression and severity of the symptoms are dependent on extra ovarian factors. Our knowledge about the actual mechanisms of PCOS and its relation to IR is indistinct. In this study, we hypothesized that IR and the associated hyperinsulinemia may play a key role in the pathophysiology of this syndrome.

Clinical evaluation of patients in the present study revealed increased BMI compared with controls that harmonized with previous investigators who report that obesity could be found in $10-50 \%$ of women with PCOS who are having BMI outside the acceptable rang of $19-25 \mathrm{~kg} / \mathrm{m}^{2(17)}$. Obesity (the fat mass), body fat location and muscle mass all have important independent effect on insulin sensitivity. Alterations in these parameters could potentially contribute to insulin resistance in PCOS in a number of ways. In particular, several metabolites (such as free fatty acid and lactate) as well as tumor necrosis factor-1 and leptin, whose production rates increases in obesity state, directly affect the peripheral action of insulin (impair insulin-mediated glucose uptake in skeletal muscle, adipocytes and liver and decrease 
hepatic insulin sensitivity and increase hepatic glucose output $)^{(\mathbf{1 9 - 2 1 )}}$.

Significant higher fasting insulin and glucose levels with decreased fasting $G / I$ ratio were observed in PCOS patients of this study when compared with control value. These results are in accordance with previous studies which reported that hyperinsulinemia due to insulin resistance occurs in the majority of women with $\operatorname{PCOS}^{(\mathbf{2 2 , 2 3 )}}$. This is thought to be due to decreased insulin sensitivity with post-receptor defect (reduced autophosphorylation of insulin receptor in the ovary) with decreasing insulin - stimulated glucose uptake ${ }^{(3)}$.

In women with PCOS, basal insulin secretion is increased and hepatic insulin clearance is reduced, resulting in hyperinsulinemia. Insulinstimulated glucose utilization is decreased by $35-40 \%$ in women with PCOS, therefore blood glucose levels become raised, a phenomenon known as hyperglycemia ${ }^{\mathbf{2 4 , 2 5})}$. Under normal circumstances, insulin secretion increases as insulin sensitivity decreases, in order to maintain glucose homeostasis. This relationship of insulin sensitivity and insulin secretion known as the deposition index that described by Pasquali et al. ${ }^{(26)}$.

In OGTT, blood glucose levels were proved to be significantly higher in PCOS patients in the existing investigation than control group and when fasting glucose levels were analyzed according to the recommendations of the Expert Commitiee of the American Diabetes Association $^{(\mathbf{1 4})}$, we found that most of PCOS patients had impaired fasting glucose $(\geq 110 \mathrm{mg} / \mathrm{dl}$ and $<126 \mathrm{mg} / \mathrm{dl})$. These results indicated the high prevalence of glucose intolerance in PCOS women, and these patients are at increased risk of developing non insulin dependent diabetes mellitus (NIDDM) that harmonized with other previous studies ${ }^{(26-28)}$.

\begin{tabular}{ccc} 
A significant & \multicolumn{2}{c}{ negative } \\
correlation between & BMI and
\end{tabular}
fasting $\mathbf{G} / \mathbf{I}$ ratio. This could support the possibility that obesity in women with PCOS can exacerbate insulin resistance and enhance the risk of NIDDM which is accounted in this study by the high prevalence of impaired oral glucose tolerance test

In the current study, PCOS patients showed significant higher levels of total testosterone, $\mathrm{LH}$ and IGF-1 compared with controls. Also in PCOS women, it was found that total testosterone is negatively correlated with fasting glucose/insulin ratio which means that the higher the insulin, the higher the androgen level suggesting a possible causal relationship. The pathophysiological mechanisms of hyperandrogenemia found in this study could be explained by that high circulating insulin levels resulting from insulin resistance stimulate ovarian theca cell androgen production both by a direct action on the ovary (through binding to insulin or IGF-1 receptors) and by stimulating the release of LH. Hyperinsulinemia may also enhance the bioavailability of androgen by decreasing the biosynthesis of IGF- binding protein-1 thus elevating IGF-1 levels which like insulin stimulates ovarian androgen production. In turn, hyperandrogenemia may increase insulin resistance in adipose tissues 
and skeletal muscle, resulting in a vicious circle of increasing androgen levels and worsening insulin resistance ${ }^{(\mathbf{2 9}, \mathbf{3 0})}$. These data support the hypothesis that insulin resistance could play a role in the pathophysiology of reproductive abnormalities characteristic of PCOS.

In the present study, ultrasound examination of the ovaries revealed significantly higher number of antral follicle in the patients group versus controls. These follicles are arrested in development at a size of 2$9 \mathrm{~mm}$, with enlarged stromal volume $>10 \mathrm{cc}$. It is suggested that excess androgens probably play a key role in the etiology of this abnormal ovarian morphology as evident by increasing levels of testosterone in PCOS patients of this study. Androgens encourage the development of primary follicles to the stages of pre-antral and small antral follicles and in the presence of excess androgen; this process is accelerated compared with normal ovary. In addition, several factors that inhibit the endogenous action of Follicle Stimulating hormone(FSH) such as follistatin, epidermal growth factor and a number of anti-apoptotic factors lead to arrest of further follicular growth and slow down the turn over of these arrested follicles. All these factors combine to give the characteristic polycystic ovarian morphology $^{(31)}$. In accordance with Bayrak et al. ${ }^{(15)}$ data of the current study showed a significant negative correlation between $\mathrm{G} / \mathrm{I}$ ratio and AFC which support that insulin resistance and hyperandrogenism have a significant impact on polycystic ovarian morphology. Further, it was reported that insulin resistance is more severe in women with PCOS who are an-ovulatory than in those who are equally hyperandrogedemic but have normal cycles. This suggests that insulin resistance contributes to anovulation $^{(32)}$.

Perhaps, the most convincing evidence linking insulin resistance with reproductive and metabolic abnormalities in women with PCOS is the utilization of insulin-sensitizing drug (metformin) to restore ovulation and metabolic disorders. Therefore, in the present study, metformin therapy improved the measured parameters of IR demonstrated by significant increase $G / I$ ratio and significant decrease total testosterone level, LH, IGF-1, AFC and BMI compared with pretreatment values. These results are in accordance with many studies $^{(33-35)}$. It was reported that metformin reduce insulin resistance and insulin secretion followed by a reduction of ovarian androgen production. Also, a direct action of metformin on ovarian theca cells to reduce androgen production was also postulated ${ }^{(36,37,9)}$.

Improved IR by metformin may not only result from the well-known reduction of hepatic glucose production and increase of peripheral glucose utilization but also from a direct effect on ovarian steroidogenesis, as demonstrated by in vitro studies of Tosca et al. ${ }^{(38)}$. Pasquali et al. ${ }^{\text {(26) }}$ found that metformin effects occur without significant action on beta-cell insulin production but may be reduced insulin response to an oral glucose load ${ }^{(39)}$.

The significant decrease in AFC observed in this study following 
metformin treatment was in agreement with Bayrak et al. ${ }^{(15)}$, however whether this effect was due to an improvement in IR is unclear, but plausible. It is also, observed that metformin as a mono-agent was capable of improving menstrual frequency and restoring ovulation in PCOS patients who have oligo/anovulation ${ }^{(\mathbf{4 0 , 4 1 )}}$. Therefore, this dose of metformin may be sufficient to achieve improvements in the biochemical markers and polycystic ovarian morphology as evident by our study. On the other hand, other studies that assessed metformin effects in hyperandrogenic subjects did not confirm these findings ${ }^{\mathbf{1 8})}$.

Laparoscopic ovarian drilling had been selected to induce ovulation in PCOS women. Although the effectiveness of bilateral ovarian wedge resection had been established, laparoscopic ovarian drilling with diathermy or LASER had been recognized as more useful because of their lower invasiveness and decrease risk of adhesions ${ }^{(7)}$. In the present work, it was found that both AFC and testosterone, LH and IGF-1 levels significantly decreased also blood glucose levels (in oral glucose tolerance test) slightly decreased and fasting $G / I$ ratio was slightly increased but the BMI was non significantly changed in PCOS patients following ovarian drilling compared with pretreatment values. These results agreed with the report conducted by Youssef and Atallah ${ }^{(\mathbf{4 2})}$ and Badawy et al. ${ }^{(43)}$ who found that in women with PCOS following ovarian drilling, there was significant improvement of ovulation and pregnancy associated with decreasing serum LH and testosterone levels but not FSH or LH / FSH ratio. Therefore, the present data observed after ovarian drilling are of considerable interest, however they need further study to clear their detail mechanisms involved.

\section{CONCLUSION}

IR plays a vital pathophysiological role in PCOS patients in term of increase BMI, T, IGF-1 and AFC but decrease G/I ratio. Also, this study suggests a possible causal relationship between insulin resistance and the reproductive and metabolic changes. Metformin and ovarian drilling potentially improve the clinical, metabolic and reproductive features of IR in PCOS women. Further investigations are needed to evaluate the cellular and molecular mechanisms of insulin resistance in PCOS.

\section{REFERENCES}

1. Wisniewski M.,Petersen M.: Is metformin beneficial for treatment of infertility in women with PCOS?.JAAPA;22:4950,2009 .

2. Azziz R, Woods KS, Reyna R, Key TJ, Knochenhauer ES and Yildiz BO. The prevalence and features of the polycystic ovary syndrome in an unselected population. Journal of Clinical Endocrinology and Metabolism; 89: 2745-2749, 2004.

3. Setji T.L. and Brown A. J.: Polycystic ovary syndrome: Diagnosis and Treatment. Am. J. Med.; 120: 128-132, 2007. 
4. Dunaif A. Insulin action in the polycystic ovary syndrome. Endocrinology and Metabolism Clinics of North America; 28 341-359, 1999.

5. Mor E, Zograbyan A, Saadat, Bayrak A. The insulin resistant subphenotype of polycystic ovarian syndrome: clinical parameters and pathogenesis. Am J Obstet Gynecol; 190:1654-60, 2004.

6. Diamanty, K. E.: Role of obesity and adiposity in polycystic ovary syndrome. Int $J$ Obes 2:S8-13, 2007.

7. De Leo V., Musacchio M.C., Morgante G., La Marca A., Petraglia F.: Polycystic ovary syndrome and type 2 diabetes mellitus. Minerva Ginecol.; 56:53-62, 2004.

8. Mathur R., Alexander CJ.,Yano J.,Trivax B. and Azziz R.:Use of metformin in polycystic ovary syndrome. Am J Obestet Gynecol, 199:596-609; 2008.

9. Tan S., Hahn S., Sven B., Tiina D., Harald L., Lars C. M., Markus S., Sigrid E., Rainer K., Klaus $M$. and Onno E J.: Metformin improves polycystic ovary syndrome symptoms irrespective of pre-treatment insulin resistance. European Journal of Endocrinology, 157: 669-676, 2007.

10. Mrazguia $\mathrm{C}$, Chevalier $\mathrm{N}$, El Fekih C. Effects of drilling on ovarian volume and follicle count in polycystic ovary syndrome. Tunis Med.; 85: 485-9, 2007.

11. Trolle B, A. Flyvbjerg ${ }^{2}$, U. Kesmodel $^{3}$ and F.F. Lauszus.
Efficacy of metformin in obese and non-obese women with polycystic ovary syndrome: a randomized, double-blinded, placebo-controlled cross-over trial. Human Reproduction, 22 2967-2973; 2007.

12. Rotterdam ESHRE/ASRMSponsored PCOS Consensus Workshop Group Revised 2003 consensus on diagnostic criteria and long-term health risks related to polycystic ovary syndrome. Fertil Steril 81:19-25, 2004.

13. Mosteller, S.A simplified calculation of body surface area. N.Engl.J.Med. 317, 1098-1112., 1987.

14. Gabir MM, Hanson RL, Dabelea D, Imperatore G, Roumain J, Bennett PH and Knowler WC.The 1997 American Diabetes Association and 1999 World Health Organization criteria for hyperglycemia in the diagnosis and prediction of diabetes. Diabetes Care; 23: 1108-1112, 2000.

15. Bayrak, A.; Terble, H.; Eliron, M.; and Paulson, R.: Acute effects of metformin therapy include improvement of insulin resistance and ovarian morphology. Fertility and Sterility, 87: 213-217; 2007.

16. Knapp G.R. and Miller M.C. Tests of statistical significance: Regression and Correlation. In: Clinical Epidemiology and Biostatistics $1^{\text {st }}$ Edition. Williams and Wilkins, Baltimore, Maryland. PP: 255 - 274, 1992. 
17. Kahan B.B. and Flier J.S.: Obesity and insulin resistance. $J$ Clin. Invest, 106: 473-481, 2000.

18. Krysiak K. R., Okapien B., Cidula-Dymec $K$. and Herman Z.: Update on the management of polycystic ovary syndrome. Pharmacol. Rep., 58: 614-625, 2006.

19. Roden M., Price T. B. and Preseghin G.: Mechanism of free fatty acid-induced insulin resistance in human. $J$ Clin. Invest, 97: 2859- 2865, 1996.

20. Poretsky L., Cataldo, N., Rosenwaks $\mathbf{Z}$. and Giudice $\mathbf{L}$.: The insulin related ovarian regulatory system in health and disease. Endocrine Rev.; 20:535582, 1999.

21. Kalra K. A.; Nair, S. and Rai. L.: Association of obesity and insulin resistance with dyslipidemia in Indian women with polycyctic Ovarian Syndrome. Indian J Med Sci., 60; 447-453, 2006.

22. Park K.H., Kim J.Y., Ahn C.W. and Song Y.D.: Polycystic Ovarian Syndrome (PCOS) and insulin resistance. Int. J. Gyn. Obstet., 74: 261-267, 2001.

23. Setji T.L. and Brown A. J.: Polycystic ovary syndrome and type 2 Diabetes. In: Feinglos M.N., Bethel M. A., eds. Type 2 Diabetes Mellitus: An evidencebased approach to practical management. Totowa, New Jersey: Humana Press; 2006.

24. O'Meara NM, Blackman DA, And Ehrmann RB. Defects in beta cell function in functional ovarian hyperandrogenism. $J$
Clin. Endocrinol and Metabol; 76:1241-1247, 1993.

25. Yildiz BO, Gedik O. Assessment of glucose intolerance and insulin sensitivity polycystic ovary syndrome. Reproductive Biochemical online; 8: 649-56, 2004

26. Pasquali R., Pelusi C., Ragazzini C. and Hasanof R.: Glucose tolerance, insulin secretion and insulin sensitivity in polycystic ovary syndrome. JOP, 3: 1-7, 2002.

27. Norman R.J., Masters L., Milner C.R. and Davies M.S.: Relative risk of conversion from normoglycemia to impaired glucose tolerance or non-insulin dependant diabetes mellitus in polycystic ovarian syndrome. Human Reproduction, 16: 19951998, 2001.

28. Legro R.S., Gnatok C.L., Kunselman A.R., and Dunaif A.: Changes in glucose tolerance over time in women with polycystic ovary syndrome: A controlled study. J. Clin. Endocrinol. Metab., 90: 32363242. 2005.

29. Zakur, H.,A.: Polycystic Ovarian Syndrome, hyperandrogenism and insulin resistance. Obestet. Gynacol. Clin. North Am., 28: 2133. 2001.

30. Livingstone, $C$. and Collison, M.: Sex steroids and insulin resistance. Clinical Science, 102: 151-166, 2003.

31. Homburg R. Polycystic ovary syndrome. Basic Practice and Research Clinical Obstetrics and Gynaecology, 22: 261-274, 2008. 
32. Ojaniemi M. and Pugeat, M.: An adolescent with polycystic ovary syndrome. Eur. $J$. Endocrinology, 155: Suppl. 1: 5149-5152, 2006.

33. Kocak M, Caliskan E, Simsir C, Haberal A: Metformin therapy improves ovulatory rates, cervical scores, and pregnancy rates in clomiphene citrate-resistant women with polycystic ovary syndrome. Fertil Steril 77:101106, 2002.

34. Hahn S, Quadbeck B, Elsenbruch S, Gartner R, Finke R, Mann K \& Janssen OE.: Metformin, an efficacious drug in the treatment of polycystic ovary syndrome. Deutsche Medizinische Wochenschrift; 129: 1059-1064, 2004.

35. Palomba S, Orio F, Falbo A, Manguso F, Russo T, Cascella T, Tolino A, Carmina E, Colao A \& Zullo F: Prospective parallel randomized, double-blind, double-dummy controlled clinical trial comparing clomiphene citrate and metformin as the firstline treatment for ovulation induction in nonobese anovulatory women with polycystic ovary syndrome. Journal of Clinical Endocrinology and Metabolism; 90: 4068-4074, 2005.

36. Fleming $\mathbf{R}$, Hopkinson ZE, Wallace AM. Ovarian function and metabolic factors in women with oligomenorrhea treated with metformin in a randomized double blind placebo-controlled trial. J Clin Endocrinol Metab; 87:569-574,2002.
37. Tang T, Glanville J, Hayden CJ: Combined lifestyle modification and metformin in obese patients with polycystic ovary syndrome. A randomized placebo-controlled double blind multicentre study. Hum Reprod; 21:80-89, 2006.

38. Tosca L, Solnais $P$, Ferre $P$, Foufelle $\mathbf{F} \&$ Dupont $J$. Metformin-induced stimulation of adenosine 5' monophosphateactivated protein kinase (PRKA) impairs progesterone secretion in rat granulosa cells. Biology of Reproduction, 75 342-351, 2006.

39. Moghetti P., Castello R., Negri C. and Tosi F., Metformin effects on clinical features, endocrine and metabolic profiles, and insulin sensitivity in polycystic ovarian syndrome: A randomized, double-blind, placebo-controlled 6-month trial, followed by open long-term clinical evaluation. J. Clin. Endocrinol. Metab., 85: 139-146. 2000.

40. 40-Lord J. M., Flight I. H. K. and Norman R. J.: Insulin sensitizing drugs (Metformin, Trogletazone, Piogletazone, DChiro-inositol) for polycystic ovary syndrome. Cochrane Database Syst. Rev., 3: CD003053, 2003.

41. 41-Moll E, Bossuyt PMM, Korevaar JC. Ovulation induced in women with newly diagnosed polycystic ovary syndrome. in a randomized double blind clinical trial comparing clomiphene citrate plus metformin with clomiphene citrate and placebo. BMJ;332:1485-91,2006. 
42. 42-Youssef $H$ and Atallah MM. Unilateral ovarian drilling in polycystic ovarian syndrome: a prospective randomized study. Reprod Biomed Online; 15:45762, 2007.

43. 43-Badawy A, Khiary M, Ragab A, Hassan M, Sherief L.
Ultrasound-guided transvaginal ovarian needle drilling (UTND) for treatment of polycystic ovary syndrome: A randomized controlled trial. Fertil Steril; 13: 135-180, 2008. 


\section{علاقة مقاومة الأنسولين و متلازمة المبيض عديد التحوصل (تأثير علاج الميتفورمين و تثثيب المبيض عليض )}

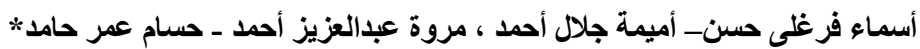

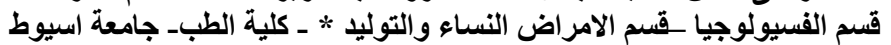

مقدمة: تعتبر متلازمة المبيض عديد التحوصل من أعقد إختلالات الغدد الصماء و الأيض والتى يكون مقاومـة

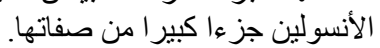

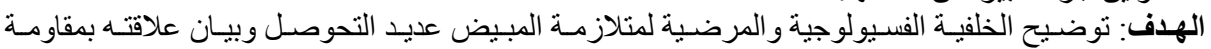

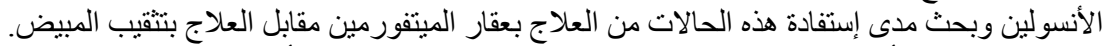

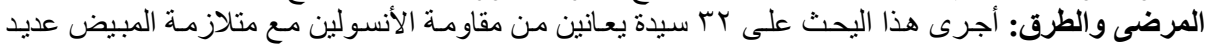

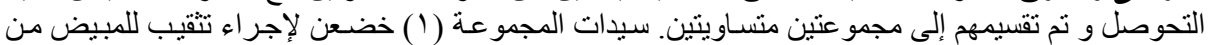

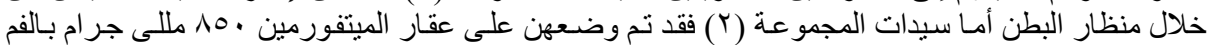

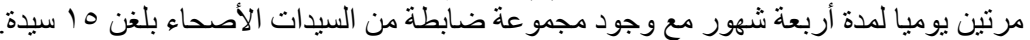

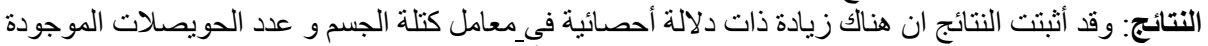

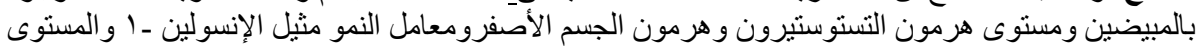

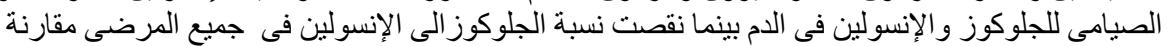

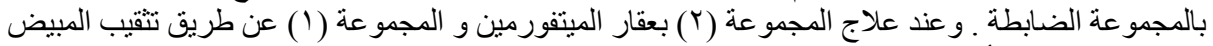

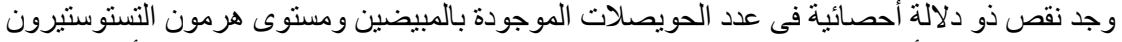

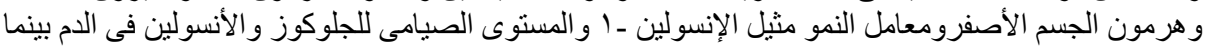

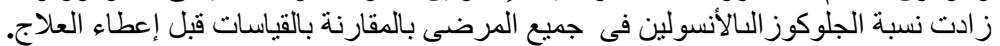

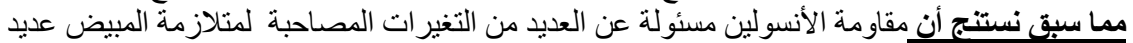

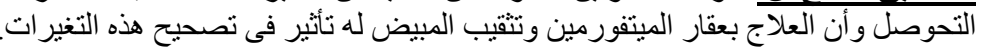

